[10] 1. E. Ackermann and B. R. Barmish, "Robust Schur stability of a polytope of polynomials," IEEE Trans. Auromat. Contr., vol. 33. pp. 984-986. 1988.

[11] B. R. Barmish, "A generalization of Kharitonov's four polynomial concept for robust stability problems with linearly dependent coefficient perturbations. "IEEE Trans. Automat. Contr., vol, 34. pp. 157-165, 1989

[12] C. B. Soh, C. S. Berger, and K. P. Dabke, "On the stability properties of polynomials with perturbed coefficients," IEEE Trans. Automat, Contr., vol. AC-30. mials with perturbed coet

[13] S. Bialas and J. Garloft, "Convex combinations of stable polynomials," J Franklin Inst, vol. 319. pp. 373-377, 1985.

[14] B. R. Barmish, "Invariance of the strict Hurwitz property for polynomiat with perturbed coefficients," IEEE Trans. Automat Contr., vol. AC-29. PP. 935-936, 1984.

[15] B. D. O. Anderson, E. I. Jury, and M. Mansour, "On robust Hurwitz polynomials." IEEE Trans. Automat. Contr., vol. AC-32, pp. 909-913, 1987.

\section{Quadratic Stability with Real and Complex Perturbations}

\section{ANDY PACKARD AND JOHN DOYLE}

Abstract - In the context of quadratic stability, the distinction between complex and real norm bounded, linear fractional perturbations is artificial, and the quadratic stability notions are equivalent to an $H_{\infty}$ norm condition on the transfer function that the perturbation "sees." In this note, we show that these connections are not true when the uncertainty is structured.

\section{INTRODLCTION}

Our notation is standard. $\boldsymbol{C}^{n \times m}$ and $\boldsymbol{R}^{r \times m}$ are, respectively, the set of complex and real $n \times m$ matrices. For $M \in C^{n \times n}$, the maximum singular value of $M$ is denoted by $\bar{\sigma}(M) . M^{*}$ is the complex conjugate transpose of $M$. For square matrices, $\rho(\cdot)$ is the spectral radius. I $M=M^{*}$, then all the eigenvalues of $M$ are real, and the notation $\lambda_{\max }(M)$ is clear.

Let $n$ and $m$ be positive integers, and suppose $M \in C^{(n-m) \times(n-m)}$, partitioned as

$$
M=\left[\begin{array}{ll}
M_{11} & M_{12} \\
M_{21} & M_{22}
\end{array}\right]
$$

where $M_{11} \in C^{n \times n}, M_{12} \in C^{n \times m}, M_{21} \in C^{m \times n}$, and $M_{22} \in C^{n \times n}$. Let $\Delta$ be a prescribed closed, convex subset of $C^{m \times m}$, which contains the origin, and define the norm bounded set $B \Delta:=\{\Delta \in \Delta: \bar{\sigma}(\Delta) \leq 1\}$ Suppose that for each $\Delta \in \boldsymbol{B} \Delta, I-\boldsymbol{M}_{22} \Delta$ is invertible, and define the linear fractional transformation $F_{l}(M, \Delta)$ as

$$
F_{l}(M, \Delta)=M_{11}+M_{12} \Delta\left(I-M_{22} \Delta\right)^{-1} M_{21} .
$$

The subscript $/$ refers to the lower block of $M$ being closed with the matrix $\Delta$, as shown in the figure below.

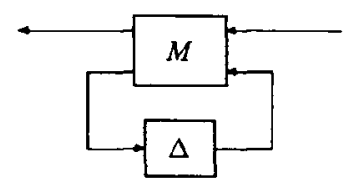

Manuscript received November 28. 1988. This work was supported by Honeywel SRC, AFOSR, ONR, and NASA.

A. Packard is with the Department of Electrical and Computer Engineering, University of California. Santa Barbara, CA 93106

J. Doyle is with the Department of Electrical Engineering, California Institute of Technology, Pasadena, CA 91125 .

IEEE Log Number 8932837.
For $\Omega \in C^{n \times n}$ with $I-M_{11} \Omega$ invertible, $F_{u}(M, \Omega)$ denotes the resultant matrix when the upper loop of $M$ is closed by $\Omega$ $F_{4}(M, \Omega):=M_{22}+M_{21} \Omega\left(I-M_{11} \Omega\right)^{-1} M_{12}$. Typically, we will use this notation when dealing with frequency responses.

Let $\left\{\Delta_{k}\right\}_{k-0}^{\boldsymbol{x}} \subset \boldsymbol{B} \boldsymbol{\Delta}$ be given, along with an initial condition $x_{0} \in \boldsymbol{C}^{n}$, define $x_{k} \in \mathcal{C}^{n}$ by

$$
x_{k-1}=F_{l}\left(M, \Delta_{k}\right) x_{k} .
$$

In this formulation, the matrix $M_{11}$ is assumed to be the nominal state space model, and $\Delta_{k} \in \boldsymbol{B} \boldsymbol{\Delta}$ is viewed as a norm bounded perturbation from an allowable perturbation class $\Delta$. The matrices $M_{12}, M_{21}$, and $M_{22}$ reflect prior knowledge on how the unknown perturbation affects the nominal dynamics $M_{11}$. This type of uncertainty, called linear fractional, is natural for many control problems, and encompasses many other special cases considered by researchers.

Definition 1.1: The pair $(M, \Delta)$ is quadratically stable if there exists a $P \in C^{n \times n}$, with $P=P^{*}>0$, such that

$$
\max _{\Delta \in B \Delta} \lambda_{\max }\left(\left[F_{l}(M, \Delta)\right]^{*} P F_{l}(M, \Delta)-P\right)<0 .
$$

Remark 1.2: The definition simply implies that there is a single. quadratic Lyapunov function $V(x):=x^{*} P x$ that establishes the stability of the entire set

$$
\left\{F_{i}(M, \Delta): \Delta \in B \Delta\right\} .
$$

In this case, regardless of $\Delta_{k} \in \boldsymbol{B} \boldsymbol{\Delta}$, as the uncertain system in (1.3) evolves, the Euclidean norm of $P^{\frac{1}{3}} \boldsymbol{x}_{k},\left\|\boldsymbol{P}^{\frac{1}{2}} \boldsymbol{x}_{k}\right\|_{2}$ decreases by at least a factor of $\beta<1$ every time step $k$, and hence robustness with respect to time-varying perturbations is guaranteed

Definition 1.3: The pair $(M, \Delta)$ is robustly stable if

$$
\max _{\Delta \in B \Delta}: \rho\left[F_{l}(M, \Delta)\right]=\beta<1 .
$$

Remark 1.4: This condition implies that for unknown, but fixed (with respect to $k$ ) uncertainty $\Delta \in \boldsymbol{B} \boldsymbol{\Delta}$. the uncertain system described by (1.3) is exponentially stable. In general, this would imply nothing about the stability of the time-varying uncertain system, where the uncertain element $\Delta$ is allowed to vary with $k$.

If the set $\Delta$ is $C^{m \times m}$, then several notions are equivalent. This is summarized in the following theorem, which is essentially found in [8]

Theorem 1.5: Let $M \in C^{(n+m) \times(n+m)}$ be given, with $\rho\left(M_{11}\right)<1$ and $\bar{\sigma}\left(M_{22}\right)<1$. Then the following are equivalent.

1) The pair $\left(M, C^{m \times m}\right)$ is quadratically stable.

2) The pair $\left(M, C^{m \times m}\right)$ is robustly stable

3) $\max _{\bar{\sigma}} \bar{\sigma}\left[F_{u}\left(M, e^{j \theta} I_{n}\right)\right]<1$.

Remark 1.6: For unstructured block uncertainty, the small gain condition [condition 3)] is a necessary and sufficient condition for robus stability to complex perturbations, and is also equivalent to quadratic stability with respect to those perturbations.

When $M$ is in fact real, an additional condition, involving real (besides complex) uncertaintics, is true. Versions of this result are found in [6], [9], and [4]

Theorem 1.7: Let $M \in R^{(n+m) \times(n+m)}$ be given, with $\rho\left(M_{11}\right)<1$ and $\bar{\sigma}\left(M_{22}\right)<1$. Then the following are equivalent.

1) The pair $\left(M, R^{m \times m}\right)$ is quadratically stable

2) The pair ( $\left.M, C^{m \times m}\right)$ is quadratically stable.

3) The pair $\left(M, C^{m \times m}\right)$ is robustly stable.

4) $\max _{\theta} \bar{\sigma}\left[F_{u}\left(M, e^{j \theta} I_{n}\right)\right]<1$.

Remark 1.8: When the data (i.c., $M$ ) arc real, the small gain theorem is a necessary and sufficient condition for robust stability with complex perturbations, and in fact is equivalent to quadratic stability with either real or complex uncertainties

Remark 1.9: Note that the norm condition [condition 4)] is an norm on the transfer function that the perturbation "sees." This the orem shows that $\|\cdot\|_{\propto}$ design techniques can be used to synthesize quadratically stable systems, and that there is equivalence between the two notions. 
Theorem 1.7 states that there is a single Lyapunov function establishing the stability of the set of matrices $\left\{F_{l}(M, \Delta): \Delta \in\right.$ $\left.\boldsymbol{R}^{m \times m}, \bar{\sigma}(\Delta) \leq 1\right\}$ if and only if every element of the set $\left\{F_{I}(M\right.$, $\left.\Delta): \Delta \in C^{m \times m}, \bar{\sigma}(\Delta) \leq 1\right\}$ is an exponentially stable matrix. This suggests some possible underlying similarity between time-varying real perturbations and fixed complex perturbations when general perturbations arise in a linear fractional manner. In the next section, we formalize two conjectures along these lines, and show that, unfortunately, neither are true.

\section{Conjectures and Counterexamples}

We begin with a definition of a structured, block diagonal uncertainty set, as introduced in [2]. Let $\boldsymbol{F}$ be either the complex. or real field. Suppose $s$ and $f$ are nonnegative integers, and positive integers $r_{1}, r_{2}, \cdots, r_{s}, m_{1}, m_{2}, \cdots, m_{f}$ are specified with $\sum_{i=1}^{s} r_{i}+\sum_{j-1}^{f} m_{j}-$ $m$. Define a set of block diagonal matrices over the field $F$ as

$\Delta_{F}:=\left\{\operatorname{diag}\left[\delta_{1} I_{r_{1}}, \cdots, \delta_{s} I_{r_{s}}, \Delta_{1}, \cdots, \Delta_{f}\right]:\right.$

$$
\left.\delta_{i} \in F, \Delta_{j} \in F^{m, \times m,}\right\} \subset F^{m \times m} . \quad(2.1)
$$

The question this section addresses is as follows. "If $\boldsymbol{M} \in \boldsymbol{R}^{(n+m) \times(n+m)}$. and a certain property is true for the pair $\left(M, \Delta_{R}\right)$, can any other property be automatically attributed to the pair $\left(M, \Delta_{C}\right)$, or vice versa?"

Theorem 1.7 above shows that when $s=0, f=1, m_{1}=m$. and the property is quadratic stability, then the two pairs share the property simultaneously. We explore this notion further when the uncertainty is structured as in (2.1)

Conjecture 2.I: Suppose $M$ is real, and $\Delta_{F}$ is a perturbation structure. If the pair $\left(M, \Delta_{R}\right)$ is quadratically stable, then the pair $\left(M, \Delta_{C}\right)$ is robustly stable.

This can be shown false with a simple counterexample. Let $-1<\beta<1$ and define $M \in \boldsymbol{R}^{3 \times 3}$ as

$$
M:=\left[\begin{array}{rrr}
0 & -\beta & \beta \\
-1 & 0 & \beta \\
-1 & \beta & 0
\end{array}\right] .
$$

This matrix $M$ comes from [7], where it was used in a slightly different context. Define $\Delta_{R}$ and $\Delta_{C}$ as

$$
\begin{aligned}
& \boldsymbol{\Delta}_{R}:=\left\{\operatorname{diag}\left[\delta_{1}, \delta_{2}\right]: \delta_{i} \in \boldsymbol{R}\right\} \\
& \boldsymbol{\Delta}_{C}:=\left\{\operatorname{diag}\left[\delta_{1}, \delta_{2}\right]: \delta_{i} \in C\right\} .
\end{aligned}
$$

For $\Delta \in \Delta_{R}$ or $\Delta \in \Delta_{C}, F_{l}(M, \Delta)$ is

$$
F_{l}(M, \Delta)=\frac{\beta\left(\delta_{1}-\delta_{2}\right)}{1-\beta^{2} \delta_{1} \delta_{2}}
$$

First, we show that this system is quadratically stable when considering real perturbations. If $\delta_{1}, \delta_{2} \in \boldsymbol{R}$, with $\left|\delta_{i}\right| \leq 1$. then

$$
\left|F_{l}(M, \Delta)\right| \leq\left|\frac{2 \beta}{1+\beta^{2}}\right|<1,
$$

therefore, the function $V(x)=x^{T} x$ is a suitable Lyapunov function that works for all $\Delta \in \boldsymbol{B} \Delta_{R}$. However, by choosing $\delta_{1}=j$ and $\delta_{2}=-j$, the quantity $F_{f}(M, \Delta)$ is

$$
F_{l}(M, \Delta)=\frac{2 \beta j}{1-\beta^{2}}
$$

which by choosing $\beta$ close enough to 1 (or -1 ) is arbitrarily large, and hence the uncertain system is not stable for fixed $\Delta \in \boldsymbol{B} \boldsymbol{\Delta}_{C}$. Hence, the conjecture is false.

Conjecture 2.2: Suppose $\boldsymbol{M}$ is real, and $\Delta_{F}$ is a perturbation structure. If the pair $\left(M, \Delta_{C}\right)$ is robustly stable, then the pair $\left(M, \Delta_{R}\right)$ is quadratically stable.

This is also false. Define $\Delta_{R}$ and $\Delta_{C}$ as in the above counterexample. Let constants $a$ and $\beta$ be chosen so $-1<a<1,-1<\beta<1$, and define
$M$ as

$$
M:=\left[\begin{array}{cccc}
a & 0 & 2 \beta a & 0 \\
0 & -a & 0 & -2 \beta a \\
0 & 1 & 0 & \beta \\
1 & 0 & \beta & 0
\end{array}\right] .
$$

For $\Delta$ in either $\boldsymbol{B} \boldsymbol{\Delta}_{R}$ or $\boldsymbol{B} \boldsymbol{\Delta}_{C}$, the linear fractional transformation $F_{/}(M, \Delta)$ is well defined, and is given by

$$
F_{\prime}(M, \Delta)=\frac{1}{1-\beta^{2} \delta_{1} \delta_{2}}\left[\begin{array}{cc}
a\left(1+\beta^{2} \delta_{1} \delta_{2}\right) & 2 \beta a \delta_{1} \\
-2 \beta a \delta_{2} & -a\left(1+\beta^{2} \delta_{1} \delta_{2}\right)
\end{array}\right] .
$$

It is straightforward to calculate that the eigenvalues of $F_{l}(M, \Delta)$ are $\pm a$, independent of the values of $\left|\delta_{1}\right|<1$ and $\left|\delta_{2}\right| \leq 1$. Hence, the pair $\left(M, \Delta_{C}\right)$ is robustly stable. However, consider the following timevarying real perturbation. For $k$ even, define $\Delta_{k}=\operatorname{diag}[1,0]$, and for $k$ odd, define $\Delta_{k}=\operatorname{diag}[0,-1]$. Then the two-step evolution of $x_{k}$ is governed by

$$
x_{k+2}=\left[\begin{array}{cc}
a^{2}\left(1+4 \beta^{2}\right) & -2 \beta a^{2} \\
-2 \beta a^{2} & a^{2}
\end{array}\right] x_{k}
$$

for $k$ odd. The eigenvalues of this matrix are at $a^{2}\left(2 \beta^{2}+1 \pm 2 \beta \sqrt{\beta^{2}+1}\right)$. As long as $\beta \neq 0, a$ can be chosen close enough to 1 so that one of these eigenvalues is bigger than 1 . Hence, the system is not even stable for time-varying real perturbations, and then certainly the pair $\left(M, \Delta_{R}\right)$ is not quadratically stable.

In fact, for any $\epsilon>0$, and $|\beta| \in(0,1)$, the constant $a$ can be properly chosen in $(-1,1)$ so that the real, time-varying perturbation $\Delta_{\text {cver }}:=\operatorname{diag}[\epsilon, 0]$ and $\Delta_{\text {odd }}:=\operatorname{diag}[0,-\epsilon]$ is destabilizing (in spite of the fact that constant perturbations from the set $B \boldsymbol{\Delta}_{C}$ are not destabilizing).

The above counterexample has another interpretation, which is fairly intuitive from a frequency domain point of view. For notational purposes, first write the uncertain difference equation $x_{k+1}=F_{l}\left(M, \Delta_{k}\right) x_{k}$ as

$$
\begin{gathered}
x_{k+1}=M_{11} x_{k}+M_{12} w_{k} \\
v_{k}=M_{21} x_{k}+M_{22} w_{k} \\
w_{k}=\Delta_{k} v_{k} .
\end{gathered}
$$

If $I-M_{22} \Delta_{k}$ is invertible, then for each $x_{k}$, there exist unique vectors $x_{k+1}, v_{k}$, and $w_{k}$ solving (2.5). Now, if we partition $x, \Delta, w$, and $v \in C^{2}$ as

$$
\left[\begin{array}{l}
\dot{x}_{k} \\
\tilde{x}_{k}
\end{array}\right]:=x_{k}, \quad\left[\begin{array}{ll}
\hat{\alpha}_{k} & 0 \\
0 & \tilde{\delta}_{k}
\end{array}\right]:=\Delta_{k}, \quad\left[\begin{array}{l}
\hat{w}_{k} \\
\tilde{w}_{k}
\end{array}\right]:=w_{k},\left[\begin{array}{l}
\hat{v}_{k} \\
\bar{v}_{k}
\end{array}\right]:=v_{k}
$$

and use the definition of $M$ in (2.3), the state equations are written explicitly as

$$
\begin{gathered}
\dot{x}_{k+1}=a \hat{x}_{k}+2 \beta a \hat{w}_{k} \\
\bar{v}_{k}=\dot{x}_{k}+\beta \hat{w}_{k} \\
\tilde{x}_{k+1}=-a \bar{x}_{k}-2 \beta a \tilde{w}_{k} \\
\hat{v}_{k}=\bar{x}_{k}+\beta \bar{w}_{k} \\
\hat{w}_{k}=\hat{\delta}_{k} \bar{v}_{k} \\
\bar{w}_{k}=\bar{\delta}_{k} \bar{v}_{k} .
\end{gathered}
$$

In terms of $\mathcal{Z}$ transforms, the time-invariant, zcro statc descriptions of (2.6) and (2.7) can be written as

$$
\begin{aligned}
& \tilde{v}(z)=T(z) \hat{w}(z) \\
& \hat{v}(z)=B(z) \dot{w}(z)
\end{aligned}
$$


where

$$
T(z)=\beta \frac{z+a}{z-a}, \quad B(z)=\beta \frac{z-a}{z+a} .
$$

Abusing notation. these equations are shown pictorially below.

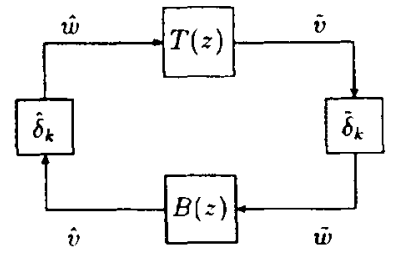

At low frequency $(z=1), T$ has a gain of $\beta(1+a) /(1-a)$. Hence. the constant input sequence $\dot{w}_{k}:=r$ will produce the constant output $\bar{v}_{k}-r \beta(1+a) /(1-a)$ [by proper choice of initial condition-in the above state representation this would be $\left.\hat{x}_{0}:=2 r \beta a /(1-a)\right]$.

Likewisc, at high frequency $(z=-1), B$ has a gain of $\beta(1+a) /(1-$ $a)$, so an initial condition of $\hat{x}_{0}:=2 t \beta a /(1-a)$ and input sequence $\bar{w}_{k}:=(-1)^{k+1} t$ will produce the output sequence $\hat{v}_{k}=\left[(-1)^{k-1} t \beta(1+\right.$ a)] $/(1-a)$.

It then follows that if the initial conditions are chosen with $r=$ $\neq 0$, and $\delta_{k}=\bar{\delta}_{k}=\left[(-1)^{k \cdot 1}(1-a)\right] / \beta(1+a)$. then the loop equations (2.6), (2.7), and (2.8) are solved, the signals $w$ and $v$ are as described above, and the state $x_{k}$ does not decay to zero. Note that for a arbitrarily close to 1 , the norm of this destabilizing perturbation $\Delta_{k}$ is arbitrarily small. In this example, the time-varying real perturbations shift the frequency content of the signals that they multiply. The subsystems $T$ and $B$ have large $(\beta(1+a) /(1-a))$ gains in different frequency ranges, therefore, the varying perturbations need only to couple these large gains together by appropriately shifting the frequency content of the signals to cause instability. Since the gains of the subsystems are large, the necessary gain of the perturbation is small. With constant perturbations, be they real or complex, this frequency shifting is impossible, and stability is assured.

III. $\|\cdot\|_{\infty}$ and Block Diagonal Quadratic Stagility

In this section we explore what connections between $\|\cdot\|_{x}$ and quadratic stability exist when the uncertainty is structured as in (2.1). recalling the strong connection for unstructured uncertainty (Theorems 1.5 and 1.7 ).

In an excellent paper [1] necessary and sufficient conditions are given for quadratic stability of the pair $(M, \Delta)$, where $M \in R^{(n+m) \times(n-m)}$, and $\Delta$ is of the form

$$
\Delta:=\left\{\operatorname{diag}\left[\delta_{1}, \delta_{2}, \cdots, \delta_{m}\right]: \delta_{i} \in \boldsymbol{R}\right\} .
$$

To state their result, define the finite set $A_{\mathrm{v}}$

$$
A_{1,}:=\left\{F_{i}(M, \Delta): \Delta \in \Delta, \delta_{i}= \pm 1\right\} .
$$

Note that $A_{v}$ has $2^{m}$ elements, and is the image, under $F_{l}(M, \Delta)$, of the vertices of $\boldsymbol{B \Delta}$. The theorem from [1] is as follows.

Theorem 3.I: Given $M$ and $\Delta$ as above. The pair $(M, \Delta)$ is quadratically stable if and only if there is a single quadratic Lyapunov function establishing the stability of the finite set of matrices $A_{v}$.

The problem of finding a single quadratic Lyapunov function for a finite set of matrices is then reformulated as a convex optimization, and the solution is discussed completely in [1]. Some synthesis results for state feedback problems with real uncertainty and quadratic stability objectives are found in [10].

For complex uncertainties, it is simpler to relate quadratic stability to a scaled $\|\cdot\|_{\infty}$ norm. We begin with a lemma about constant scaling of transfer functions.

Lemma 3.2: Let $\mathscr{D} \subset C^{m \times m}$ be a set of invertible matrices. Define

$\bar{D}:=\left\{\operatorname{diag}[T, D]: T \in C^{n \times n}, T=T^{*}>0, D \in \mathbb{D}\right\}$

$$
\subset C^{(n+m) \times(n-m)} \text {. }
$$

Let $M \subset C^{(n+m) \times(n+m)}$ be given, with $\rho\left(M_{11}\right)<1$. Then, there exists a $D \in D$ with

$$
\max _{\theta \in|0,2 \pi|} \bar{\sigma}\left[D F_{u}\left(M, e^{j \theta} I_{n}\right) D^{-1}\right]<1
$$

if and only if

$$
\inf _{\bar{D} \in D} \bar{\sigma}\left(\tilde{D} M \tilde{D}^{-1}\right)<1
$$

Proof: This follows from the results in [1] or [5].

Next, consider a specific block structure $\Delta$ of the form

$$
\Delta:=\left\{\operatorname{diag}\left[\Delta_{1}, \Delta_{2}\right]: \Delta_{1} \in C^{m_{1} \times m_{1}}, \Delta_{2} \in \boldsymbol{C}^{m_{2} \times m_{2}}\right\} \subset \boldsymbol{C}^{m \times m}
$$

where $m_{1}+m_{2}=m$. Define $\mathfrak{D}$ and $\mathfrak{D}$ as

$$
\begin{gathered}
\mathscr{D}:=\left\{\operatorname{diag}\left[d I_{m_{1}}, I_{m_{2}}\right]: d>0\right\} \subset \boldsymbol{R}^{n \times m} \\
\hat{D}:=\left\{\operatorname{diag}\left\{d_{1} I_{n}, D\right]: d_{1}>0, D \in \mathscr{D}\right\} \subset \boldsymbol{R}^{(n+m) \times(n+m)}
\end{gathered}
$$

and $\tilde{D}$ as in (3.1).

Assume that for all $\Delta \in \boldsymbol{B} \Delta$, the matrix $I-M_{22} \Delta$ is nonsingular (this would be satisfied if and only if $\mu_{\Delta}\left(M_{22}\right)<1$, where $\mu_{\Delta}\left(M_{22}\right)$ is the structured singular value of $M_{22}$ with respect to the structure $\Delta$ [2]. Then the following lemma is equivalent to a main result of [2], and is discussed further in [5].

Lemma 3.3:

$$
\max _{\lrcorner \in B \Delta} \bar{\sigma}\left[F_{l}(M, \Delta)\right]<1 \text { if and only if } \inf _{D \in D} \bar{\sigma}\left(\hat{D} M \hat{D}^{-1}\right)<1 .
$$

Lemma 3.4: Consider the set $\Delta$ as defined in (3.2), and corresponding $D$ in $(3.3)$. The pair $(M, \Delta)$ is quadratically stable if and only if there is a $D \in \mathbb{D}$ such that

$$
\max _{\theta \in[0,2 \pi]} \bar{\sigma}\left[D F_{4}\left(M, e^{j \theta} I_{n}\right) D^{-1}\right]<1 .
$$

Remark 3.5: Using the structured singular value theory developed in [2], it is possible to show that the pair $(M, \Delta)$ is robustly stable if and only if for each $\theta \subseteq[0,2 \pi]$, there is a $D_{\theta} \in \mathbb{D}$ such that $\bar{\sigma}\left[D_{\theta} F_{u}\left(M, e^{j \theta} I_{n}\right) D_{\theta}^{-1}\right]<1$. This is a weaker condition, since the scaling matrix can change with frequency $\theta$ while the condition in Lemma 3.4 involves a constant scaling matrix.

Proof: For each $T \in C^{n \times n}, T=T^{*}>0$, define $M_{T} \in$ $C^{(n+m) \times(n+m)}$ by

$$
M_{T}:=\left[\begin{array}{cc}
T M_{11} T^{-1} & T M_{12} \\
M_{21} T^{-1} & M_{22}
\end{array}\right] .
$$

Now the pair $(M, \Delta)$ is quadratically stable if and only if there is a $T \in C^{n \times n}$, with $T=T^{*}>0$, such that

$$
\max _{\Delta \in B \Delta} \bar{\sigma}\left[T F_{l}(M, \Delta) T^{-1}\right]=\max _{\Delta \in B \Delta} \bar{\sigma}\left[F_{l}\left(M_{T}, \Delta\right)\right]<1 .
$$

Using Lemma 3.3 , this is true if and only if there exists a $T$ such that

$$
\inf _{\dot{D} \in \mathfrak{s}} \bar{\sigma}\left(\hat{D} M_{T} \hat{D}^{-1}\right)<1
$$

which is equivalent to

$$
\inf _{\bar{D} \in \bar{D}} \bar{\sigma}\left(\tilde{D} M \tilde{D}^{-1}\right)<1 .
$$

This is the same condition as in Lemma 3.2.

Hence, for two full complex blocks, the notion of quadratic stability is equivalent to a $\|\cdot\|_{\infty}$ criterion, with constant, diagonal scaling matrices. The equivalence for more than two blocks is in general not true, since the result in Lemma 2.3 exploits the fact that $\Delta$ has only two blocks.

\section{Conclusions}

In this note we have shown that the equivalence between real and complex perturbations in the context of quadratic stability to linear fractional, 
unstructured perturbations is not true when the perturbations are block structured. For a limited class of problems, quadratic stability in the face of structured complex perturbations is equivalent to scaled $\|\cdot\|_{\infty}$ norms, and hence $\|\cdot\|_{x}$ synthesis techniques, coupled with diagonal constant scalings, can be used to design quadratically stable systems.

\section{Acknowledgmeni}

The authors would like to acknowledge K. Zhou, P. Khargonekar, and K. Poolla for helpful discussions.

\section{REFERFNCES}

[1] S. Boyd and Q. Yang. "Structured and simultaneous Lyapunov functions fur system stability problems," Inform. Syst. Lab. Stanford Univ. Tech. Rep. L104-88-1, Mar. 1988: also in Int. J. Contr., 1989.

[2] J. C. Doyle. "Analysis of feedback systems with structured uncertainties," IEE Proc., vol. 129, pan D, no. 6. pp. 242-250, Nov. 1982

[3] J. C. Doyle and A. Packard, "Uncerain multivariable systems from a state spacc perspective." in Proc. Amer. Contr. Conf., June 1987, pp. 2147-2152.

[4] P. Khargonekar, I. Petersen, and K. Zhou, IEEE Trans. Automat. Contr., submitted for publication.

[5] A. Packard and J. Doyle. "Structured singular value with repeated scalar blocks." in Proc. Amer. Contr. Conf, Atlanta. GA. June 1988, pp. 1213-1218.

16] V. M. Popov, "Absolute stability of nonlinear systems of automatic control," Automat. Remote Contr., vol. 22. pp. 857-875, 1962

[7] S. Skogestad, personal communication.

[8] J. C. Willems, "Least squares stationary optimal control and the algebraic Riccati equation," IEEE Trans. Automat Contr., vol. AC-16, no. 6. pp. 621-634, Dec. 1971 .

[9] J. L. Willems, "The circle criterion and quadratic Lyapunov functions for stability analysis." IEEE Trans. Automat. Contr. vol. AC-18, p. 184, Apr. 1973.

[10] J. Bernussou, P. L. D. Peres, J. C. Geromel. "A linear programming oriented procedure for quadratic stabilization of uncertain systems," Syst. Conir. Lett. vol. 13, no. 1, pp. 65-72, 1989

\section{On the Norms Used in Computing the Structured Singular Value}

\section{NAM-KIU TSING}

Abstract-Different norms are considered to replace the Euclidean norm in an algorithm given hy Fan and Tits (IEEE Trans. Automat. Contr., vol. 33, pp. 284-289, 1988) which is used for the computation of the structured singular value of any matrix. It is shown that the $l_{1}$ norm is the best possible norm in a certain sense.

Recently, there has been a considerable amount of interest in the study of the structured singular value, the concept of which was originated by Doyle [1] and is used as a tool for the analysis and synthesis of feedback systems with structured uncertainties (e.g., see [2], [3] and their references).

Let $M$ be an $n \times n$ complex matrix, and $\pi=\left(k_{1}, \cdots, k_{m}\right)$ an $m$ tuple of positive integers which satisfies $\sum_{i=1}^{m} k_{i}=n$. For $i=1, \cdots, m$, denote the $i$ th-block-projection matrix by $P_{i}=$ block diag $\left(O_{k_{1}}, \cdots, O_{k_{1-1}}, I_{k_{1}}, O_{k_{-1}}, \cdots, O_{k_{m}}\right)$ where $O_{k}$ and $I_{k}$ are the zero matrix and identity matrix, respectively, of order $k \times k$ for any positive integer $k$. Then the structured singular value of $M$ with respect to the block structure $\mathfrak{K}$ is the nonnegative scalar

$$
\mu(M)=\max _{x \in i^{\prime \prime} B}\left\{\|M x\|:\left\|P_{1} x\right\|\|M x\|=\left\|P_{i} M x\right\|, i=1, \cdots, m\right\}
$$

where $\|\cdot\|$ denotes the Euclidean $\left(l_{2}\right)$ norm in $G^{\prime \prime}$ and $\partial B$ the corresponding unit sphere. One major issue in the study of $\mu(M)$ is the computation of it. In their paper [2], the authors devise an algorithm [2, Algorithm 11, which we shall explain immediately, to compute $\mu(M)$. They first

Manuscript received March 3, 1988: rcvised April 11, 1988 and Novernber 15, 1988. This work was supported by the National Science Foundation's Engineering Research Centers Program NSF CDR 8803012 and by the National Science Foundation under Grant DMC-84-51515.

The author is with the Systems Research Center and the Department of Electrical Engineering. University of Maryland, College Park, MD 20742

IEEE Log Number 8931894 define, for any real number $\alpha$, the Hermitian matrices

$$
A_{i}(\alpha)=\alpha P_{i}-M^{H} P_{i} M, \quad \text { for } i=1, \cdots, m
$$

and the $m$-form numerical range associated with $A_{1}(\alpha), \cdots, A_{m}(\alpha)$ :

$W(\alpha)=\left\{\left(v_{1}, \cdots, v_{m}\right) \in\right\}^{m}: \exists x \in \partial B$

such that $v_{i}=x^{H} A_{i}(\alpha) x$ for all $\left.i\right\}$.

A function $c(\cdot): \Omega \rightarrow s$, which depends on $M$ and $\Re$, is then defined by

$$
c(\alpha)=\min \{\|v\|: v \in W(\alpha)\}
$$

where $\|\cdot\|$ again denotes the Euclidean norm (on $\$^{m}$ this time). Then they show [2, Corollary 1 and Proposition 1] that, for any matrix $M$ with structure $\mathfrak{K}, c(\cdot)$ satisfies

$$
c(\cdot) \text { is continuous }
$$

$$
c\left(\mu^{2}\right)=0, c(\alpha)>0 \quad \text { for all } \alpha>\mu^{2}
$$

and

$$
c(\alpha+s)<c(\alpha)+s \quad \text { for all } s>0 \text { and real } \alpha
$$

where $\mu=\mu(M)$. The initial step in [2, Algorithm 1] is to set $\alpha_{1 j}=$ $\bar{\sigma}^{2}(M)$ where $\bar{\sigma}(M)$ is the largest singular value of $M$. The iteration step is to set $\alpha_{k+1}-\alpha_{k}-c\left(\alpha_{k}\right)$ for $k=0,1,2, \cdots$. The authors show in $[2$, Theorem 2] that, since $c(\cdot)$ satisfies (1)-(3), Algorithm 1 will generate a monotonic decreasing sequence $\left\{\alpha_{k}\right\}$ with $\lim _{k} \rightarrow \infty \alpha_{k}=\mu^{2}$. The structured singular value $\mu$ can thus be obtained. They also remark in the footnote that the Euclidean norm in the definition of $c(\cdot)$ can be replaced by the $l_{1}$ norm to get the strongest version of Proposition 1 . The purpose of the present note is to elaborate on this remark.

Suppose we consider any norm $N(\cdot)$ on $s s^{m}$ instead of the Euclidean norm. Similar to the case of $c(\cdot)$, we may define a function $\left.\left.c_{N}: s\right\} \rightarrow ;\right\}$ (which also depends on $M$ and $\mathcal{K}$ ) by

$$
c_{N}(\alpha)=\min \{N(v): v \in W(\alpha)\} .
$$

It is not hard to see that $c_{N}(\cdot)$ always satisfies (1) and (2); and if in addition $c_{N}(\cdot)$ satisfies (3) also, then the function $c(\cdot)$ in Algorithm 1 can be replaced by $c_{N}(\cdot)$. Let

\section{$\Re=\left\{N(\cdot): N(\cdot)\right.$ is a norm on $\boldsymbol{s}^{m}$ and $c_{N}(\cdot)$ satisfies}

condition (3) for all matrices $M$ with structure $\mathfrak{K}$ \}.

Then Algorithm 1 will work with $c(\cdot)$ being replaced by any $c_{N}(\cdot)$ where $N(\cdot) \in \mathfrak{\pi}$. In view of the iteration step of the algorithm, we may want to choose a norm $N_{0}(\cdot) \in \Re$ such that

$$
c_{N_{11}}(\alpha) \geq c_{N}(\alpha) \quad \text { for all } N(\cdot) \in \mathfrak{N} \text { and } \alpha>\mu^{2}
$$

so that the resulting algorithm has the fastest convergent rate and is thus the most efficient. The following result shows that $H_{1} H_{1}$, i.e., the $t_{1}$ norm on $\mathrm{s}^{\mathrm{m}}$ defined by

$$
\|v\|_{1}=\sum_{i-1}^{m}\left|v_{i}\right| \quad \text { for all } v=\left(v_{1}, \cdots, v_{m}\right) \in E 3^{m},
$$

will give such a "best possible" norm.

Theorem: Let $\mathfrak{K}=\left(k_{1}, \cdots, k_{m}\right)$ be a given block structure. Then

a) $\|\cdot\|_{1} \in \mathfrak{I}$, and

b) for any $N(\cdot) \in \mathscr{R},\|v\|_{1} \geq N(v)$ for all $v \in \mathrm{s}^{\prime \prime}$, so that

$$
c_{1}(\alpha):=\min \left\{\|v\|_{1}: v \in W(\alpha)\right\} \geq c_{N}(\alpha)
$$

for any real $\alpha$ and complex matrix $M$ with block structure $\Re$. Proof:

a) The proof of the fact that $c_{1}(\cdot)$ satisfies (3) is similar to (and 